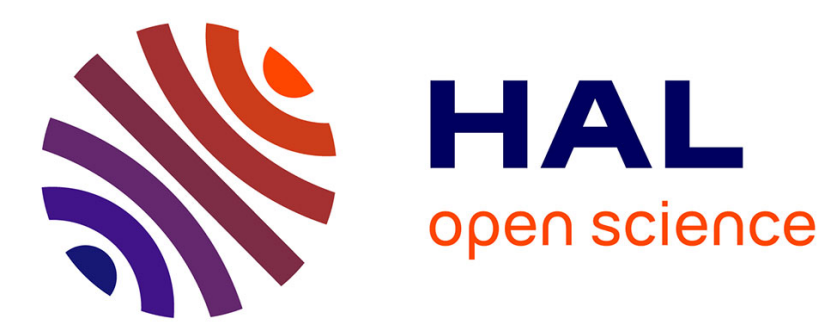

\title{
Non-invasive Optimal Coupling Upon Detection of a Local Change of Impedance in a Cable Network
}

\author{
K. Brahima Yeo, Matthieu Davy, Philippe Besnier
}

\section{To cite this version:}

K. Brahima Yeo, Matthieu Davy, Philippe Besnier. Non-invasive Optimal Coupling Upon Detection of a Local Change of Impedance in a Cable Network. 2021 IEEE International Joint EMC/SI/PI and EMC Europe Symposium, Jul 2021, Glasgow, United Kingdom. hal-03576186

\author{
HAL Id: hal-03576186 \\ https://hal.science/hal-03576186
}

Submitted on 15 Feb 2022

HAL is a multi-disciplinary open access archive for the deposit and dissemination of scientific research documents, whether they are published or not. The documents may come from teaching and research institutions in France or abroad, or from public or private research centers.
L'archive ouverte pluridisciplinaire HAL, est destinée au dépôt et à la diffusion de documents scientifiques de niveau recherche, publiés ou non, émanant des établissements d'enseignement et de recherche français ou étrangers, des laboratoires publics ou privés. 


\section{Non-invasive Optimal Coupling Upon Detection of a Local Change of Impedance in a Cable Network}

\author{
K. Brahima Yeo \\ Univ Rennes, INSA Rennes, CNRS, \\ IETR-UMR 6164, \\ F-35000 Rennes, France \\ kyeo@insa-rennes.fr
}

\author{
Matthieu Davy \\ Univ Rennes, CNRS, \\ IETR-UMR 6164, \\ F-35000 Rennes, France \\ matthieu.davy@univ-rennes1.fr
}

\author{
Philippe Besnier \\ Univ Rennes, INSA Rennes, CNRS, \\ IETR-UMR 6164, \\ F-35000 Rennes, France \\ philippe.besnier@insa-rennes.fr
}

\begin{abstract}
In this paper, we apply a novel wavefront shaping technique within a cable network. By manipulating an array of crosstalk sources at different locations of the network, we demonstrate experimentally a strong enhancement of the intensity at a specific wire end where an impedance change occurs. The optimal wavefront for maximal focusing is determined noninvasively using the generalized Wigner-Smith operator. Our approach relies on two successive measurements of the scattering matrix measured at the injection ports, before and after the change. The optimal wavefront then closely corresponds to the first eigenstate of the generalized Wigner-Smith operator. Thus, a maximum intensity may be reached at a remote distance without cooperation of the target, opening up new perspectives in the context of electromagnetic aggressions or attacks (cybersecurity).
\end{abstract}

\section{INTRODUCTION}

Intentional Electromagnetic Interference (IEMI) is nowadays a well known threat for various electronic equipment, systems or infrastructures. Many different strategies may be used to cause dysfunctions, including severe ones that would end as a denial of service (DoS). These strategies involve conducted as well as radiated interference, continuous (modulated) narrow band signals or wide, ultra-wide band signals, as explored in [1]. A particular problem is that of defining appropriate protections to cope with these attacks. Standardization offers a certain protection level against standardized attacks but attackers may develop their own strategies according to their technological expertise and imagination [2]. This a major concern regarding advanced digital technologies within smart grids [3], wire communications [4] (introducing repetitive crosstalk disturbances), wireless communications [5] [6], autonomous vehicles [7]. From the point of view of risk analysis or attack optimization, evaluation of worse or more favorable configurations are also the subject of questions. Optimization of the coupling of radiated IEMI on a cable was proposed in [8]. A possibly successful strategy consists in using phase conjugation or its wide-band analog, the time reversal (TR) technique, to enhance wire or wireless coupling to the targeted victim [9]. However, these techniques require some kind of cooperation from the targets since they require the knowledge of the impulse response between the transmitter (source of aggression) and the receiver (the victim). Recent techniques of wavefront shaping may overcome this hurdle as further illustrated in this communication.
Indeed, new approaches relying on measurement of the scattering matrix $S(\omega)$ have recently emerged to overcome the need of measuring the vector of transmission coefficients to the target. The scattering matrix $S(\omega)$ provides the field transmission and reflection coefficients between incoming and outgoing channels coupled to a system. The optimal wavefront for maximal focusing on a target subjected to parametric variations can be extracted from the generalized Wigner-Smith operator (WSO). The WSO, originally known as the time delay operator, was introduced by Eugene Wigner and Félix Smith in quantum mechanics [10], [11] to study the scattering properties of particles interacting with a potential well. In this case, the WSO is related to the derivative of $S(\omega)$ with frequency. The WSO has recently been generalized to the derivation of $S(\omega)$ with any local or global parameter $\alpha$ and called the Generalized Wigner-Smith (GWS) operator [12]. Optimal focusing on scatterers that are slightly translated, rotated or subjected to a variation of the dielectric constant have been demonstrated. In the microwave domain, one can also leverage the modulation of an antenna's impedance [13] (unpublished).

In this article, we demonstrate non-invasive focusing within a cable network using the Wigner-Smith operator. To this end, we locally tune the impedance at a point of the network and show that the first eigenstate of the WSO related to the derivative of $S(\omega)$ with this local shift provides the incoming vector giving maximal intensity at this selected point. The paper is organized as follows. We first introduce the theoretical background of the WSO in Section II. Then, the experimental results with several crosstalk sources in two configurations of a cable network are provided in Section III. The first elementary configuration features the main properties of the method. The second one then extends our technique to a more realistic scenario with a complex cable network. The last section provides some conclusions and perspective.

\section{WIGNER-SMITH OPERATOR}

The Wigner-Smith time delay operator $Q$ originally involves the derivative of the $(N \times N)$ scattering matrix with respect to frequency as

$$
Q=-i S^{-1} \frac{\partial S}{\partial \omega}
$$


The $Q$-matrix generalizes to multichannel systems the singlechannel time delay provided by the phase derivative of a single transmission coefficient. A key property of its eigenstates is that they are dispersion free ; they are insensitive to small variations $\Delta \omega$ of the frequency except to a global factor given by $e^{i \Delta \omega \tau(\omega)}$, where $\tau(\omega)$ is the corresponding eigenvalue [14]. These eigenstates are hence related to well-defined time delays given by the real part of the eigenvalues. In contrast, the magnitude of the eigenvalue's imaginary parts reflects how the amplitude of the outgoing wavefront is affected by the frequency change. The left eigenvectors of $Q$ provide the incoming wavefronts to excite these states.

Recently, the $Q$ operator was generalized to the derivative of $S(\omega)$ with any global or local parameter $\alpha$. It led to the concept of the generalized Wigner-Smith (GWS) operator [12]

$$
Q_{\alpha}=-i S^{-1} \frac{\partial S}{\partial \alpha}
$$

$\alpha$ can for instance be the position of a movable scatterer. It has been demonstrated that the eigenstate of $Q_{\alpha}$ with lowest eigenvalue is insensitive to small displacements of the target so that the intensity of the field is minimal at the target location. In contrast, the eigenstate with maximal eigenvalue maximizes the intensity on the target. The corresponding eigenvector hence provides the optimal wavefront for maximal focusing.

In this communication, we aim at demonstrating that the GWS is applicable to a local change of impedance $\alpha$ in a cable network. Experimentally, we tune the impedance at the end of a wire to either $220 \Omega$ or $150 \Omega$ and measure the scattering matrices $S_{1}(\omega)$ and $S_{2}(\omega)$ between crosstalk sources in both cases. The $Q_{\alpha}$ matrix is estimated from $Q_{\alpha}=-i S_{1}^{-1}\left[S_{2}(\omega)-S_{1}(\omega)\right] . Q_{\alpha}$ is then diagonalized and we estimate the focused intensity corresponding to its eigenstates. The intensity at the target for an incoming wavefont $\psi_{\text {in }}$ is synthetically reconstructed with

$$
Y(\omega)=\left|\psi_{i n}^{T} T(\omega)\right|^{2},
$$

where the $T(\omega)$ is the $N \times 1$ vector of field transmission coefficients between the sources and the target. To demonstrate the maximization of the intensity with our non-invasive technique, $T(\omega)$ is estimated with a receiver placed at the target position. The intensity at the target for an eigenstate of $Q_{\alpha}$ is therefore $Y_{i}(\omega)=\left|q_{i}^{\dagger} T(\omega)\right|^{2}$, where $q_{i}$ is the corresponding left eigenvector. Note that $q_{i}$ is a vector with unit norm. After sorting and arranging the eigenvalues according to their absolute values $\left|\lambda_{i}\right|$, we select the maximal one and the corresponding eigenvector $q_{1}$ defines the input wavefront to reach the optimal focusing $Y_{o p t}=\left|q_{1}^{\dagger} T(\omega)\right|^{2}$.

We assess the performance of our approach by comparing the focused intensity to a reference assuming the complete channel information as if the target was a cooperative one. In this condition, the reference intensity with maximal focusing (i.e. the highest achievable performance), $Y_{r e f}$, is obtained by illuminating the network with the normalized phase-conjugate of the the transmission vector $T(\omega)$.

$$
Y_{\text {ref }}(\omega)=\left|\frac{T^{\dagger}(\omega)}{\|T(\omega)\|} T(\omega)\right|^{2}=\|T(\omega)\|^{2} .
$$

Our metric hence consists in comparing $Y_{o p t}$ to $Y_{\text {ref }}$. We also compare the focused intensity to the one obtained with random illuminations of the system. In the following, $Y_{\text {rand }}$ is the average of $\left|\psi_{\text {rand }}^{T} T(\omega)\right|^{2}$ for 1001 normalized random vectors $\psi_{\text {rand }}^{T}, Y_{\text {rand }}=\left\langle\left|\psi_{\text {rand }}^{T} T(\omega)\right|^{2}\right\rangle$.

We designed two experimental setups, both over ground planes, to first verify the efficiency of our approach in the simple case of two isolated wires (see Fig. 1) and then extend it to a more complex and realistic scenario shown in Fig. 3. The second setup combines 3 coupling sources and a set of three wires in common mode transmission over a single ground plane associated to a ground wire over all paths. In the both cases, the injections on the wires are carried out with pieces of open circuit wires, by means of crosstalk coupling. All sources of injection are connected to vector network analyser (VNA) from Agilent (N5230C). The receiver at the target (where the local change of impedance takes place) is a small current probe of type F-36-4, also connected to the VNA. Therefore, this probe enables to check for the focalization performance.

\section{EXPERIMENTS AND RESULTS}

A. Test case : wires on two separate ground planes (2 injections)

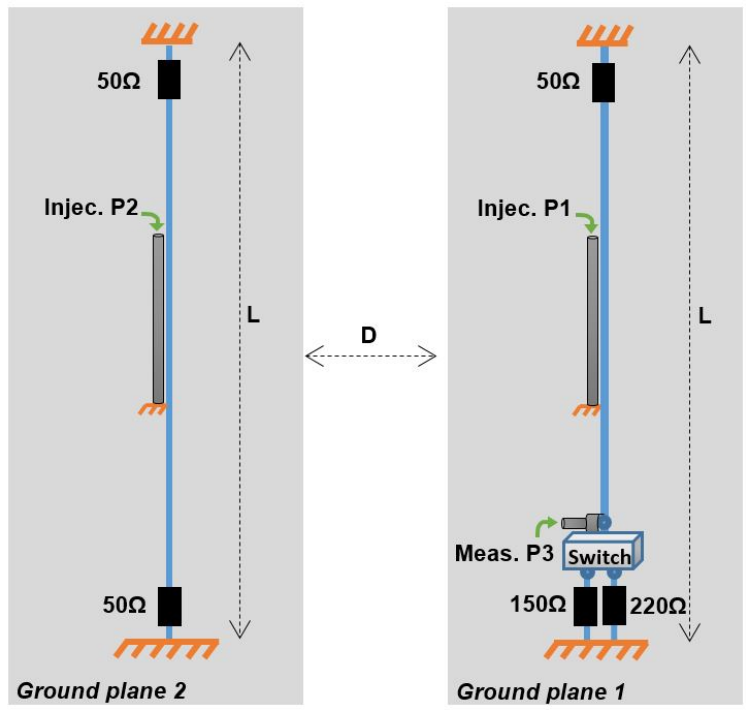

Fig. 1. Drawing of two excited wires over separate ground planes. The length of both excited wires is $L=130 \mathrm{~cm}$ and ground planes are separated from each other by $D=70 \mathrm{~cm}$. The length of the two injection wires connected to the port 1 (Injec. P1) and port 2 (Injec. P2) of the VNA is $45 \mathrm{~cm}$. The measurement probe located close to the switch is connected to the third port (Meas. P3) of the VNA .

To start with a simple experiment, we place two wires of the same length $(L=130 \mathrm{~cm})$ over two ground planes separated from each other by $D=70 \mathrm{~cm}$ as shown in Fig. 1. At both top ends and at the bottom end of the second wire, they are connected to their corresponding ground planes through $50 \Omega$ 
loads. The injections (P1 and P2) at the middle of the wires are carried out with two pieces of wire of length $45 \mathrm{~cm}$, by means of crosstalk coupling. We induce a change at the bottom end of the first wire by switching a load to either $220 \Omega$ or $150 \Omega$ with an RF coaxial switch device of type SEM123D. We then measure $S(\omega)$ for the two states of the load in the 400-800 $\mathrm{MHz}$ frequency range.

The variations with frequency of the magnitude of the two eigenvalues of the GWS operator are presented in Fig. 2(a). The first one is clearly dominating the second one over the frequency range, except around $673 \mathrm{MHz}$. Because the coupling between the ground planes is small, we expect that only the injection port $\mathrm{P} 1$ is sensitive to the impedance change so that the components of the first eigenvector of $Q_{\alpha}$ should be $\left[\begin{array}{ll}1 & 0\end{array}\right]$ for any frequency. This is generally confirmed in Fig. 2(b) with a focused intensity $Y_{\text {opt }}$ obtained by injecting the first eigenvector which closely corresponds to the maximal intensity obtained with phase-conjugation $Y_{r e f}$ (see Fig. 2(c)). An exchange between the eigenvectors is however observed near $673 \mathrm{MHz}$. In a small range, the two eigenvalues with low magnitudes are close and the $Q_{\alpha}$ matrix does not provide a reliable indicator of the wire that should be excited for focusing on the change.

From an engineering point of view, there is no question about the strategy of injection in such a simple case. However, we have verified that any random combined excitations of the two injection ports does not provide such a focusing performance. The intensity is indeed enhanced by $3 \mathrm{~dB}$ relative to its average over random excitations. From this first and simple experiment, we deduce that, for optimal focusing, the highest eigenvalue must be distinct from the other one. This must be checked prior to any injection.

The second experiment aims at confirming that several sources may be combined with appropriate complex weights to reach an optimal focusing in a more complex and realistic cable network. To do so, we designed a setup with a fourbranch cable network. Three different injection sources are now used as depicted in Fig. 3.

\section{B. Cable network over a ground plane with 3 injection sources}

In this experimental configuration, the three left branches are separated from each other by $25 \mathrm{~cm}$ and the fourth, where the target is located, is separated from the third by $30 \mathrm{~cm}$ (Fig. 3). Three wires are "live" wires in common mode configuration whereas the fourth wire plays the role of a ground wire. In fact, this ground wire (wire \#4) consists of 4 sections interconnected to a common splice. The total length of the sections is $4.65 \mathrm{~m}$. It is directly connected to the ground plane at all ends. The length of the wires \#1, \#2 and \#3 are $2.85 \mathrm{~m}, 2.65 \mathrm{~m}$, and $1.55 \mathrm{~m}$, respectively. The wires \#2 and \#3 are also connected to the ground plane at both ends through $50 \Omega$ loads. As far as the wire \#1 is concerned, it is connected to the ground plane through a $50 \Omega$ at the left end. The switch now takes place at the right hand of the wire \#1 and plays the same role as in the first experiment (automatic switching between $220 \Omega$ or a $150 \Omega$ ). Three sources with same injection
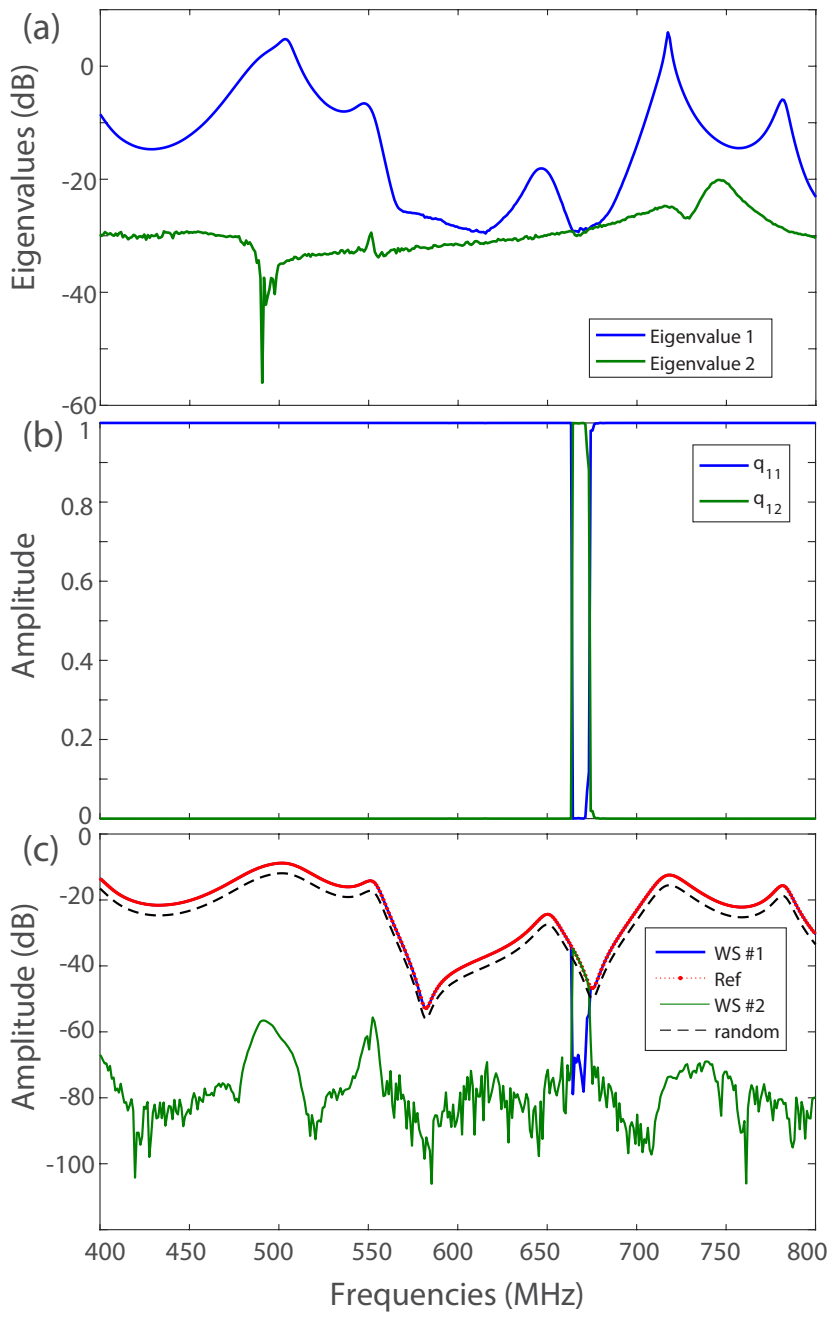

Fig. 2. (a) Variations of the magnitude of the eigenvalues of $Q_{\alpha}$ between $400 \mathrm{MHz}$ and $800 \mathrm{MHz}$. The eigenvalues shown in blue and green are sorted in decreasing order. (b) Variation of the components of the first eigenvector $q_{1}$ ( $q_{11}$ and $q_{12}$ are the first and second component, respectively). The blue and green lines correspond to its first and second components respectively. (c) Intensity found at the target for the two eigenvectors shown with blue and green colours. The blue line is hence $Y_{\text {opt }}(\omega)$. The black dash line corresponds to an average over 1001 normalized random incoming vectors, $Y_{\text {rand }}(\omega)$. The red dotted line is the reference maximal focusing $Y_{r e f}(\omega)$ obtained with the phase-conjugation technique.

means are located on the three left branches of the network. Measurements are carried out in exactly the same conditions using the same current probe as a receiver.

After measurement of the scattering matrix $S$ in the two states of the load, the three ordered (from maximum in blue color to minimum in orange color) eigenvalues of the $Q_{\alpha}$ operator are represented in the $100-500 \mathrm{MHz}$ frequency range in Fig. 4(a). The magnitude of the components of the corresponding eigenvectors shown in Fig. 4(b) highlights that the first eigenvector is a complex combination of the three sources. Fig. 4(c) confirms that when the first eigenvalue clearly dominates the second one (by for instance an order of magnitude), the first eigenvector provides an optimal focusing on the target with $Y_{\text {opt }}(\omega)$ (blue solid line) reaching $Y_{\text {ref }}(\omega)$ 


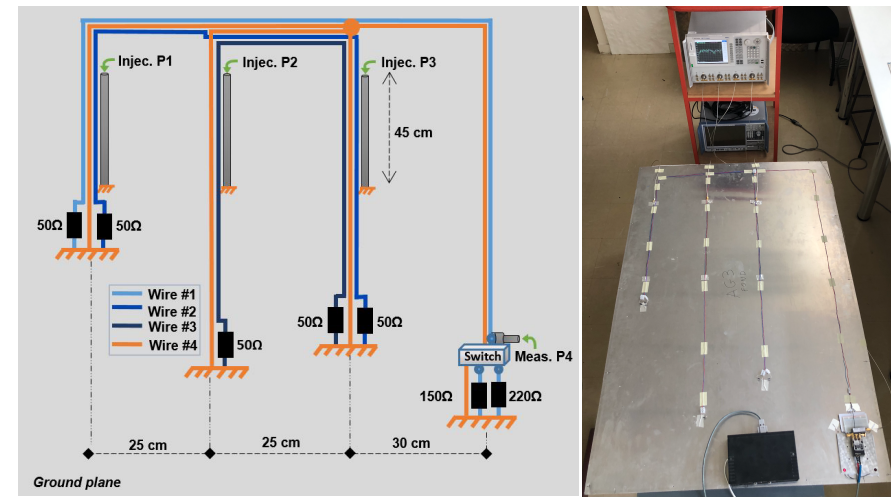

Fig. 3. Drawing of the complex wire network with three injections at the left. Picture of the experience with a VNA for injections and measurements at the right. The three antennas are connected to the ports 1 (Injec. P1), port 2 (Injec. P2) and port 3 (Injec. p3) of the VNA. The measurement clamp at the switch is connected to the port 4 (Meas. P4) of the VNA. The switch is a voltage controlled switch to change the impedance.

(red dot line) as if the complete channel information was accessible. At certain frequencies (e.g between $335 \mathrm{MHz}$ and $365 \mathrm{MHz}$ ), the optimal eigenvector even corresponds to injecting nearly all energy at the injection point which is the furthest from the target (Injec. P1).

In agreement with the observations made for the first experiment, however, when the eigenvalues are weak and cannot be clearly discriminated (for instance near $135 \mathrm{MHz}$ and $378 \mathrm{MHz}$ ), maximal focusing is not provided by the eigenvector. The coupling levels at those frequencies are generally low. We also show the intensity at the target for an average over random vectors (black dash line) as well as the second and third eigenvectors (green solid line and orange solid line). Clearly, the focused intensity is in general not even close to the one of the first eigenvector.

Eventually, this experiment demonstrate that the generalized Wigner-Smith operator is a powerful and simple tool to perform optimal coupling on a cable network without prior knowledge of the transfer function between the sources and the target. The location of the target is not required either. This operator is therefore very sensitive to an impedance change at any end of the network. For instance, detection of plug-in or plug-off operations should be achievable.

\section{CONCLUSION}

This paper presented a non-invasive approach to optimize the coupling within a cable network where a small impedance change takes place at some end of the network. The technique takes advantage of the properties of the GWS operator based on the difference between two scattering matrices measured at input ports. As soon as its eigenvalues are sufficiently distinct from each other, the energy can be maximally focused at the targeted point, as if the channel information was known. This result opens up new perspectives in the context of IEMI or cybersecurity related to electromagnetic attacks. Further developments will deal with the extension of such techniques for wideband focusing.
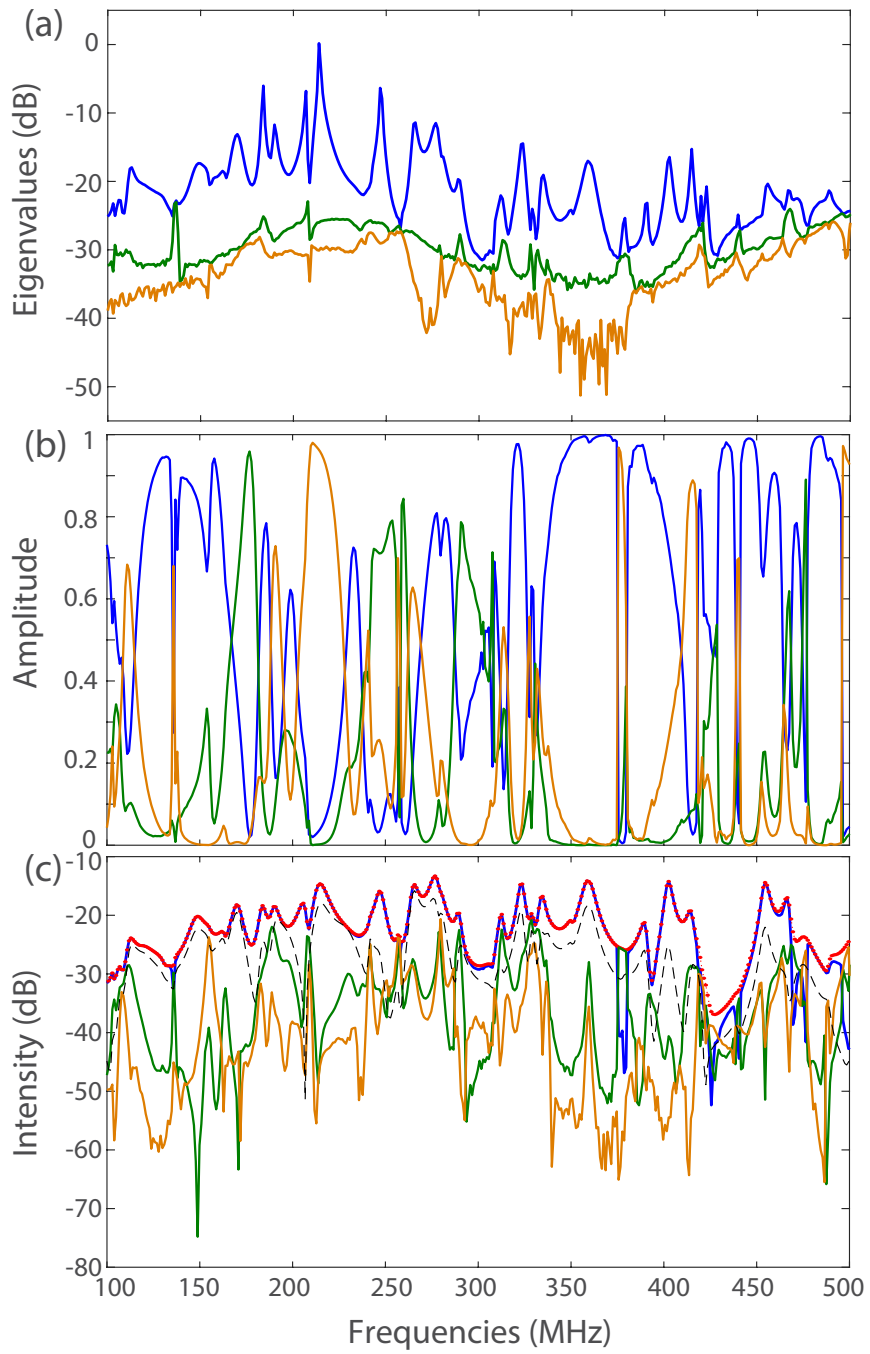

Fig. 4. Same as Fig. 2 for a more complex network with three injection sources. Measurements are here carried out between $100 \mathrm{MHz}$ and $500 \mathrm{MHz}$. In addition to the blue and green lines already shown in Fig. 2, the orange lines in $(a, b, c)$ represent the magnitude of the third eigenvalue, the third component of the first eigenvector and the intensity at the target obtained for an injection of the third eigenvector, respectively.

\section{ACKNOWLEDGMENT}

This publication was supported by the European Union through the European Regional Development Fund (ERDF), by the French region of Brittany and Rennes Métropole through the CPER Project SOPHIE/STIC \& Ondes and by the French "Agence Nationale de la Recherche" under reference ANR-17-ASTR-0017. M.D. acknowledges the "Institut Universitaire de France".

\section{REFERENCES}

[1] W. A. Radasky, C. E. Baum and M. W. Wik, "Introduction to the special issue on high-power electromagnetics (HPEM) and intentional electromagnetic interference (IEMI)", IEEE Transactions on Electromagnetic Compatibility, vol. 46, no. 3, pp. 314-321, 2004.

[2] G. Lugrin, N. Mora, S. Sliman, F. Rachidi, M. Rubinstein and R. Cherkaoui, "Overview of IEMI conducted and radiated sources: Characteristics and trends," 2013 International Symposium on Electromagnetic Compatibility, Brugge, 2013, pp. 24-28. 
[3] M. Lanzrath, M. Suhrke and H. Hirsch, "HPEM-Based Risk Assessment of Substations Enabled for the Smart Grid," in IEEE Transactions on Electromagnetic Compatibility, vol. 62, no. 1, pp. 173-185, Feb. 2020, doi: 10.1109/TEMC.2019.2893937.

[4] K. -J. Li, Y. -Z. Xie, F. Zhang and Y. -H. Chen, "Statistical Inference of Serial Communication Errors Caused by Repetitive Electromagnetic Disturbances," in IEEE Transactions on Electromagnetic Compatibility, vol. 62, no. 4, pp. 1160-1168, Aug. 2020, doi: 10.1109/TEMC.2019.2932855.

[5] J. Villain, V. Deniau, A. Fleury, E. P. Simon, C. Gransart and R. Kousri, "EM Monitoring and Classification of IEMI and Protocol-Based Attacks on IEEE 802.11n Communication Networks," in IEEE Transactions on Electromagnetic Compatibility, vol. 61, no. 6, pp. 1771-1781, Dec. 2019, doi: 10.1109/TEMC.2019.2900262.

[6] Villain, V. Deniau, C. Gransart, A. Fleury and E. P. Simon, "Characterization of IEEE 802.11 Communications and Detection of Low-Power Jamming Attacks in Noncontrolled Environment Based on a Clustering Study," in IEEE Systems Journal, doi: 10.1109/JSYST.2020.3045365.

[7] P. Ängskog, P. Näsman and L. Mattsson, "Resilience to Intentional Electromagnetic Interference Is Required for Connected Autonomous Vehicles," in IEEE Transactions on Electromagnetic Compatibility, vol. 61, no. 5, pp. 1552-1559, Oct. 2019, doi: 10.1109/TEMC.2018.2866178.

[8] T. Liang, G. Spadacini, F. Grassi and S. A. Pignari, "Coupling of Wideband Radiated IEMI to Cables Above Ground," in IEEE Transactions on Electromagnetic Compatibility, vol. 62, no. 2, pp. 589-597, April 2020

[9] M. Davy, J. de Rosny, J.C. Joly, M. Fink, "Focusing and amplification of electromagnetic waves by time reversal in an leaky reverberation chamber," Comptes Rendus Physique, vol. 11, Issue 1, pp. 37-43, 2010.

[10] F. T. Smith, "Lifetime matrix in collision theory," Physical Review, vol.118, no. 1, p. 349-356, Jan 1960.

[11] E. P. Wigner,"Lower Limit for the Energy Derivative of the Scattering Phase Shift," Phys. Rev., vol. 98, pp. 145-147, 1955.

[12] P. Ambichl, A. Brandstötter, J. Böhm, M. Kühmayer, U. Kuhl, and S. Rotter, "Focusing inside disordered media with the generalized WignerSmith operator," Phys.Rev.Lett., vol. 119, no. 3, 2017,Art. no. 033903.

[13] P. del Hougne, K. Brahima Yeo, P. Besnier, M. Davy, "Programmable meta-atom inclusions in complex media enable coherent wave control with an arbitrary wavefront," arXiv:2011.04514 [physics.app-ph], submitted October 23, 2020.

[14] S. Rotter, P. Ambichl, and F. Libisch, "Generating particle like scattering states in wave transport," Phys. Rev. Lett., vol. 106, pp. 120602 (1-4), 2011.

[15] S. Rotter and S. Gigan, "Light fields in complex media: Mesoscopic scattering meets wave control," Rev. Mod. Phys., vol. 89, p. 015005, 2017.

[16] M. Fink, "Time Reversed Acoustics," Phys. Today, vol. 50, pp. 34-40, 1997.

[17] G. Lerosey, J. de Rosny, A. Tourin, and M. Fink, "Focusing Beyond the Diffraction Limit with Far-Field Time Reversal," Science, vol. 315, pp. 1120-1122, February 23, 20072007

[18] A. P. Mosk, A. Lagendijk, G. Lerosey, and M. Fink, "Controlling waves in space and time for imaging and focusing in complex media," Nature Photon., vol. 6, pp. 283-292, 2012.

[19] J. Aulbach, B. Gjonaj, P. M. Johnson, A. P. Mosk, and A. Lagendijk, "Control of Light Transmission through Opaque Scattering Media in Space and Time," Phys. Rev. Lett., vol. 106, p. 103901, 2011. 